\title{
Estudo retrospectivo das implicações maternas, fetais e perinatais em mulheres portadoras de diabetes, em 20 anos de acompanhamento no Hospital Escola da Universidade Federal do Triângulo Mineiro
}

\author{
Retrospective study of the maternal, fetal and perinatal \\ outcomes in diabetic women in twenty years of assistance in the \\ Universidade Federal do Triângulo Mineiro School Hospital
}

Evelyne Gabriela Schmaltz Chaves', Priscila de Melo Franciscon', Gabriel Antônio N. Nascentes², Marina Carvalho Paschoini ${ }^{3}$,

Adriana Paula da Silva' ${ }^{1}$, Maria de Fátima Borges ${ }^{1}$

1 Disciplina de Endocrinologia Departamento de Clínica Médica, Universidade Federal do Triângulo Mineiro (UFTM), Uberaba, MG, Brasil 2 Departamento de Ciências Biológicas, UFTM, Uberaba, MG, Brasil ${ }^{3}$ Departamento de Ginecologia e Obstetrícia, UFTM Uberaba, MG, Brasil
Correspondência para: Maria de Fátima Borges Hospital de Clínicas,

Universidade Federal do Triângulo Mineiro, Departamento de Clínica Médica, Disciplina de Endocrinologia Rua Getúlio Guaritá s $/ n^{\circ}$ 38025-440 - Uberaba, MG, Brasil borgmf@uol.com.br

Recebido em 7/Abr/2010 Aceito em 14/Set/2010

\section{RESUMO}

Objetivos: Avaliar implicações do diabetes melito (DM) na morbimortalidade materno-fetal, segundo experiência da Universidade Federal doTriângulo Mineiro. Materiais e métodos: Procedeu-se à análise retrospectiva dos prontuários de gestantes diabéticas assistidas entre 1990 e 2009 focando dados e complicações maternas e neonatais. Resultados: A última gestação de 93 diabéticas foi avaliada, sendo 34 com DM tipo 1, em que se observou maior ocorrência de tocotrauma $(p=0,023)$ e retinopatia $(p=0,023)$. Vinte e uma pacientes tinham DM tipo 2; suas necessidades de insulina aumentaram progressivamente $(p<0,01)$ e observou-se maior prevalência de tabagismo $(p=0,004)$. Trinta e oito tiveram diabetes gestacional e iniciaram acompanhamento do diabetes em idade gestacional mais tardia $(p<0,001)$, tiveram mais antecedentes de macrossomia fetal $(p=0,028)$ e maior prevalência de fatores de risco cardiovascular. Conclusões: Não obstante melhora do controle glicêmico durante a gestação, nenhum dos grupos atingiu alvos glicêmicos ideais. Ainda assim, a maioria das gestações em diabéticas, conduzidas em nosso meio, evoluiu favoravelmente. Arq Bras Endocrinol Metab. 2010;54(7):620-9

\section{Descritore}

Diabetes melito tipo 1; diabetes melito tipo 2; diabetes gestacional; gravidez de alto risco; morbidade; neonato

\section{ABSTRACT}

Objectives: Evaluation of the consequences of diabetes mellitus (DM) on maternal and fetal morbidity and mortality at Universidade Federal do Triângulo Mineiro. Materials and methods: A retrospective review of medical records of pregnant women with diabetes was carried out at the hospital between 1990-2009, focusing on maternal and neonatal data and complications. Results: The last pregnancy of 93 diabetics was evaluated. In thirty-four patients with type 1 diabetes a higher incidence of birth trauma $(p=0.023)$ and retinopathy $(p=0.023)$ was observed. Twentyone type 2 DM subjects required progressively increased insulin therapy $(p<0.01)$ and showed a higher prevalence of smoking $(p=0.004)$. Thirty-eight had gestational diabetes. Their diabetic follow-up started at a later gestational age $(p<0.001)$, had more fetal macrosomia histories ( $p$ $=0.028$ ) and cardiovascular risk factors. Conclusions: Despite improvement of glycemic control during pregnancy neither group attained the glycemic target. However, the majority of DM pregnancies evaluated in our group presented successful outcomes. Arq Bras Endocrinol Metab. 2010;54(7):620-9

Keywords

Type 1 diabetes mellitus; type 2 diabetes mellitus; gestational diabetes mellitus; high risk pregnancy; morbidity; neonate 


\section{INTRODUÇÃO}

$\mathrm{N}$ as últimas décadas, o diabetes melito (DM) tem se tornado um sério e crescente problema de saúde pública em todo o mundo devido ao aumento de sua prevalência, morbidade e mortalidade $(1,2)$. É tradicionalmente classificado em diabetes melito do tipo 1 (DM1), tipo 2 (DM2) e gestacional (DMG). O DMl, caracterizado por deficiência de insulina, desenvolve-se mais comumente na infância e adolescência, acometendo mulheres em idade reprodutiva. O DM2, embora ocorra predominantemente em adultos, tem acometido de forma crescente indivíduos jovens. Quando diagnosticado durante a gestação, o DMG pode ou não persistir após o parto $(3)$.

Do ponto de vista obstétrico, o DM é considerado um complicador gestacional, existindo uma clara relação entre o controle glicêmico e a morbimortalidade materno-fetal (4). Apesar disso, mulheres diabéticas têm uma chance em torno de $97 \%$ a $98 \%$ de dar à luz uma criança saudável se aderirem a um programa de tratamento e supervisão cuidadosos $(5,6)$.

A morbidade em gestantes diabéticas depende de sua saúde e do controle glicêmico, principalmente no período pré e concepcional. Elas estão, de forma geral, mais sujeitas a processos infecciosos, cetoacidose diabética $(\mathrm{DMl})$, à piora de determinadas complicações crônicas do DM e à hipertensão arterial induzida pela gravidez. O tratamento envolve dieta, atividade física e tradicionalmente a insulinoterapia, embora o uso de hipoglicemiantes orais tenha sido alvo de grande interesse e de pesquisas na atualidade $(5,6)$.

É consenso entre diversos autores de que o DM representa uma importante intercorrência clínica durante a gestação, e a maior prevalência de DM2 entre mulheres em idade reprodutiva associada ao aumento da incidência de DMG nos últimos anos faz com que estudos envolvendo tais patologias no período gestacional sejam relevantes, visando reduzir a morbimortalidade materno-fetal (7).

Por intermédio deste estudo, avaliamos, do ponto de vista clínico e metabólico, gestantes assistidas pelos serviços de Endocrinologia e de Ginecologia e Obstetrícia (GO) do Hospital de Clínicas da Universidade Federal do Triângulo Mineiro (UFTM) durante o período pré, peri e pós-gestacional, traçando um paralelo entre os grupos de gestantes com DM1, DM2 e DMG, observando implicações do DM no curso da gestação e sobre a morbimortalidade da gestante e do neonato em nosso meio.

\section{MATERIAIS E MÉTODOS}

O estudo retrospectivo envolveu a revisão de prontuários de gestantes diabéticas assistidas tanto em nível ambulatorial quanto durante internação hospitalar. A avaliação considerou desde a admissão da gestante na instituição referida, prosseguindo durante a gestação até o período pós-parto, objetivando-se avaliação clínica e metabólica, assim como exame clínico e análise glicêmica dos neonatos e da morbimortalidade maternofetal. Pacientes com diagnóstico pré-gestacional de DM acompanhadas no serviço também foram avaliadas por meio de exames laboratoriais registrados nos prontuários nos três anos anteriores à atual gestação, visando à análise pré-gestacional. O período de avaliação foi de 1990 a 2009. A pesquisa obteve autorização do Comitê de Ética em Pesquisa da UFTM.

Foram incluídas gestantes com idade mínima de 18 anos portadoras de DMl (grupo 1) ou DM2 (grupo 2) que engravidaram e gestantes que desenvolveram DM durante o período gestacional (grupo 3), sendo essas pacientes acompanhadas pela equipe de Endocrinologia, sempre que solicitada pela disciplina de Ginecologia e Obstetrícia.

Foram excluídas pacientes que procuraram assistência médica apenas no momento do parto por não terem sido acompanhadas durante a gestação, aquelas cujos prontuários estavam incompletos impossibilitando a análise e portadoras de outros tipos de diabetes.

Os dados maternos avaliados foram idade cronológica, idade gestacional na consulta inicial, antecedentes pessoais e familiares relevantes, dados laboratoriais pré-gestacionais nos três últimos anos, antecedentes obstétricos, complicações crônicas do DM, hábitos e condições de vida, terapêutica instituída no início e no decorrer da gestação, intercorrências durante a gestação, dados laboratoriais, ganho ponderal, índice de massa corporal (IMC) - por meio da média trimestral dos valores encontrados, sendo a análise dos valores obtidos realizada com base na tabela para avaliação do IMC conforme a idade gestacional preconizada pelo Ministério da Saúde (8), período de internação para controle glicêmico, adequação alimentar, tipo de parto, complicações obstétricas e tempo de seguimento pós-parto.

Os neonatos foram avaliados durante o período de internação e no pós-parto imediato. Os dados analisados foram peso ao nascimento, glicemia capilar periférica e ocorrência de malformações. 
$\mathrm{Na}$ avaliação dos dados laboratoriais, foi considerada a média aritmética dos valores glicêmicos em cada trimestre (glicemia de jejum e 2 horas pós-prandial) e dos valores de colesterol total e frações, triglicérides, hemoglobina glicosilada, frutosamina, TSH e T4 livre quando registrados nos prontuários.

Para avaliação pré-gestacional de pacientes com diagnóstico de DM1 e DM2, foram resgatados dos prontuários os valores de glicemia de jejum (GJ), 2 horas pós-prandial (GPP), frutosamina, hemoglobina glicosilada, colesterol total e frações, e triglicérides de cada consulta ambulatorial nos três anos anteriores à gestação analisada e calculada a média aritmética desses valores para análise.

A avaliação da adequação alimentar foi realizada a partir dos registros encontrados nos prontuários, sendo os dados obtidos por meio de entrevista no ato do atendimento clínico de cada paciente, bem como pela avaliação dos dados antropométricos antes e durante a gestação.

Pacientes com DMl e DM2 vinham sendo acompanhadas pelo serviço de Endocrinologia do HC-UFTM, tendo diagnóstico prévio estabelecido conforme critérios preconizados pela Associação Americana de Diabetes (9); os diagnósticos de DMG também foram realizados de acordo com critérios estabelecidos por essa associação (10).

Foi considerada macrossomia fetal quando o peso do neonato ao nascimento foi maior que $4.000 \mathrm{~g}$ ou superior ao percentil 90 para a idade gestacional $(9,10)$.

Foi considerado bom controle glicêmico valores de GJ iguais ou menores que $95 \mathrm{mg} / \mathrm{dl}$ e GPP (2h) iguais ou menores que $120 \mathrm{mg} / \mathrm{dl}$. Valores acima desses níveis foram considerados como controle inadequado durante a gestação $(11,12)$.

A determinação das dosagens bioquímicas de rotina do HC-UFTM tem empregado sistemas de automação Cobas Mira e Cobas Integra 400 Plus e estojos comerciais da Roche ${ }^{\circledast}$. Concentrações de glicose foram determinadas pelo método enzimático de referência com hexoquinase, tendo como valores de referência atuais (VR) GJ entre 70 e $100 \mathrm{mg} / \mathrm{dl}$ e GPP < 140 $\mathrm{mg} / \mathrm{dl}$ (13). Para dosagem de hemoglobina glicosilada, foi usado o método de inibição turbimétrica (TINIA), sendo os VR atuais de $4,8 \%$ a $5,9 \%$, considerando-se para fins de controle glicêmico valores menores que 7\% (14). Para determinação da frutosamina, foi utilizado teste colorimétrico por reação com azul de nitrotetrazólico, tendo como VR atual 205 a $285 \mu \mathrm{mol} / 1$ (15).
Para dosagem de colesterol total, HDL-colesterol e triglicérides, foi utilizado o método colorimétrico e considerados os VR habituais (16-18); as dosagens de TSH e T4 livre foram determinadas pela imunoquimioluminescência a partir de 1994 (Immulite 2000 - Diagnostic Products Corporation, DPC ${ }^{\circledR}$, Los Angeles - Califórnia - USA), sendo os VR 0,38 a 4,5 ng/dl e 0,8 a 2,3 ng/dl, respectivamente (19).

\section{Análise estatística}

As variáveis numéricas foram inicialmente analisadas por meio do teste de Kolmogorov-Smirnov para a distribuição dos dados e pelo teste de Levene para verificação da homogeneidade das variâncias.

Nas comparações entre dois grupos independentes (variáveis com valores somente para os grupos DMl e DM2), foi utilizado o teste t-Student quando os dados apresentaram distribuição normal e homogeneidade das variâncias e o teste de Mann-Whitney quando essas condições não foram encontradas.

Nas comparações entre três grupos independentes (DMl, DM2 e DMG), utilizou-se o teste de ANOVA seguido do teste de Bonferroni, para amostras paramétricas, ou o teste de Kruskal-Wallis seguido do teste de Dunn, para amostras não paramétricas.

Nas comparações entre três amostras dependentes (primeiro, segundo e terceiro trimestre de gestação), foi utilizado o teste de ANOVA para medidas repetidas seguido do teste de Bonferroni ou o teste de Friedman seguido da diferença mínima não paramétrica.

As variáveis categóricas foram analisadas em relação aos grupos clínicos por meio do teste do qui-quadrado clássico ou, quando necessário, o teste do qui-quadrado com correção de Yates e teste exato de Fisher. A análise dos resíduos (diferença entre a frequência observada e a frequência esperada) foi utilizada para auxiliar na interpretação dos valores significativos encontrados no teste do qui-quadrado.

As análises estatísticas foram realizadas por meio do software Statistica 7.0 (StatSoft, Inc.) e as diferenças observadas foram consideradas significativas quando $o$ nível de significância $(p)$ foi menor que 0,05 .

\section{RESULTADOS}

Foram avaliados prontuários de 104 gestantes e, destes, excluídos 11, sendo cinco por estarem incompletos, três porque a paciente procurou a instituição apenas na véspera do parto e três pelo DM ser secundário à pancrea- 
tite crônica. Entre os selecionados, 34 tinham diagnóstico prévio de $\mathrm{DMl}$ (grupo 1), 21 tinham diagnóstico prévio de DM2 (grupo 2) e 38 tiveram o diagnóstico de DM durante a gestação atual (DG) (grupo 3).

Os três grupos diferiram entre si $(\mathrm{p}<0,001)$ quanto à idade, sendo que as pacientes com DMl tiveram idade materna menos avançada, mediana: 25 anos, (mínima: 20; máxima: 39) do que as portadoras de DM2, 36 anos (22 a 44). Quanto à idade gestacional (IG) na primeira consulta, as pacientes do grupo 3 apresentaram IG significativamente mais avançada $(\mathrm{p}<0,001)$, mediana: 27 semanas ( 9 a 38), em relação aos demais grupos.

Considerando os antecedentes obstétricos, não houve diferença estatisticamente significante entre os três grupos quanto à ocorrência de parto pré-termo, DMG prévio, óbito neonatal, natimortos e abortamentos prévios. Fetos macrossômicos em gestações anteriores predominaram no grupo $3(23,7 \% ; p=0,008)$ em relação aos outros grupos e pré-eclâmpsia/eclâmpsia prévia foi mais referida no grupo $2(23,8 \% ; \mathrm{p}=0,014)$ (Tabela 1$)$.

Os antecedentes pessoais relevantes referidos pelas pacientes foram representados na tabela 2. Os três grupos não diferiram quanto a dislipidemia, síndrome de ovários policísticos (SOP), etilismo, uso de drogas ilícitas e hipotireoidismo; houve predomínio de hipertensão arterial sistêmica $(\mathrm{p}=0,009)$ e sobrepeso/ obesidade $(\mathrm{p}=0,001)$ no grupo 3 ; de tabagismo $(\mathrm{p}=$ $0,004)$ e cardiopatia isquêmica $(\mathrm{p}=0,008)$ no grupo 2 e de cetoacidose diabética prévia $(\mathrm{p}=0,03)$ no grupo 1 . Tabagismo esteve presente em $23(24,7 \%)$ e etilismo em $6(6,4 \%)$ do total de pacientes.

Quanto às variáveis analisadas nos três anos pré-gestacionais, constatamos que pacientes do grupo 2 apresentavam IMC $(\mathrm{p}=0,006)$ e triglicérides $(\mathrm{p}=0,005)$ significativamente maiores que as do grupo 1 , não havendo diferença quanto às outras variáveis analisadas (GJ, GPP, frutosamina, hemoglobina glicosilada, colesterol total, LDL e HDL) (Tabela 3).

Não houve diferença significativa $(\mathrm{p}=0,068)$ quanto ao tempo prévio referido de DM entre os grupos 1 , mediana: 7 anos (1 a 18) e 2, mediana: 4 anos (1 a 6). Complicações crônicas do DM foram encontradas apenas entre pacientes do grupo 1 , sendo mais frequente a retinopatia $(\mathrm{n}=7 ; 20,6 \%)$, embora 4 pacientes $(11,8 \%)$ apresentaram nefropatia e $2(5,9 \%)$, neuropatia. Indícios de resistência insulínica, como obesidade abdominal e acantose nigricans, foram mais prevalentes $(\mathrm{p}=$ $0,035)$ no grupo 3.
Tabela 1. Dados obstétricos prévios e durante a gestação nos grupos 1, 2 e 3

\begin{tabular}{|c|c|c|c|c|c|c|}
\hline \multirow{2}{*}{ Características } & \multicolumn{2}{|c|}{ DM1 (n:34) } & \multicolumn{2}{|c|}{ DM2 (n:21) } & \multicolumn{2}{|c|}{ DMG (n:38) } \\
\hline & $\mathbf{n}$ & $\%$ & $\mathrm{n}$ & $\%$ & $\mathbf{n}$ & $\%$ \\
\hline \multicolumn{7}{|c|}{ Complicações em partos prévios } \\
\hline Fetos macrossômicos & 1(a) & 2,9 & 2(b) & 9,5 & 9(c) & 23,7 \\
\hline Pré-eclâmpsia/eclâmpsia & 0 (d) & 0,0 & $5(e)$ & 23,8 & $4(\mathbf{f})$ & 10,5 \\
\hline Parto pré-termo & 3 & 8,8 & 1 & 4,8 & 3 & 7,9 \\
\hline DMG prévio & 0 & 0,0 & 2 & 9,5 & 2 & 5,3 \\
\hline Óbito neonatal & 0 & 0,0 & 2 & 9,5 & 3 & 7,9 \\
\hline \multicolumn{7}{|c|}{ Tipo de parto (quando realizado no HC-UFTM) } \\
\hline Vaginal & 5 & 14,8 & 2 & 14,3 & 4 & 13,3 \\
\hline Cesariana & 29 & 85,1 & 12 & 85,7 & 26 & 86,7 \\
\hline \multicolumn{7}{|c|}{ Intercorrências durante a gestação } \\
\hline $\begin{array}{l}\text { Internações para controle } \\
\text { glicêmico }\end{array}$ & 21 & 61,8 & 10 & 47,6 & 21 & 55.3 \\
\hline Cetoacidose & $12(\mathbf{g})$ & 35,3 & 1 (h) & 4,8 & 0 (i) & 0,0 \\
\hline Óbito materno & 0 & 0,0 & 1 & 4.8 & 0 & 0,0 \\
\hline \multicolumn{7}{|l|}{ Complicações parto atual } \\
\hline Parto pré-termo & 10 & 29,4 & 3 & 14,3 & 6 & 15,8 \\
\hline Sofrimento fetal agudo & 5 & 14,7 & 2 & 9,5 & 5 & 13,2 \\
\hline Polidrâmnio & 3 & 8,8 & 1 & 4,8 & 4 & 10,6 \\
\hline Amniorrexe prematura & 5 & 14,7 & 1 & 4,8 & 5 & 13,2 \\
\hline Abortamento & 4 & 11,8 & 1 & 4,8 & 0 & 0,0 \\
\hline Pré-eclâmpsia/eclâmpsia & 7 & 20,6 & 3 & 14,3 & 4 & 10,6 \\
\hline Sinais de tocotrauma & $5(\mathbf{j})$ & 14,7 & $0(\mathbf{k})$ & 0,0 & $0(\mathbf{l})$ & 0,0 \\
\hline $\begin{array}{l}\text { Infecção tardia da ferida } \\
\text { operatória }\end{array}$ & $0(\mathbf{m})$ & 0,0 & $2(\mathbf{n})$ & 9,5 & $0(0)$ & 0,0 \\
\hline Distocia de ombro & 1 & 2,9 & 0 & 0,0 & 0 & 0,0 \\
\hline Insuficiência placentária & 1 & 2.9 & 0 & 0,0 & 0 & 0,0 \\
\hline
\end{tabular}

$\mathbf{a} \times \mathbf{b} \times \mathbf{c}-p=0,028 ; \mathbf{d} \times \mathbf{e} \times \mathbf{f}-\mathrm{p}=0,014 ; \mathbf{g} \times \mathbf{h} \times \mathbf{i}-p<0,001 ; \mathbf{j} \times \mathbf{k} \times \mathbf{I}-\mathrm{p}=0,023 ; \mathbf{m} \times \mathbf{n} \times \mathbf{0}$ $-p=0,03$, sendo que as frequências de $\mathbf{c}, \mathbf{e}$ e $\mathbf{g}$ foram maiores que 0 esperado, as de $\mathbf{b}, \mathbf{d}$ e $\mathbf{i}$, menores que o esperado e as de $\mathbf{j}$ e $\mathbf{n}$, com características associadas ao grupo.

Os grupos não diferiram quanto à adequação alimentar. O peso das gestantes do grupo 1 foi inferior $(\mathrm{p}<$ $0,001)$ no primeiro, segundo e terceiro trimestres, comparado aos pesos obtidos nos grupos 2 e 3 (Tabela 4).

As medianas das glicemias de jejum (GJ) no primeiro trimestre foram mais elevadas $(\mathrm{p}=0,027)$ no grupo $1,172 \mathrm{mg} / \mathrm{dl}$ (77 a 358) em comparação com os grupos $2,115 \mathrm{mg} / \mathrm{dl}(97 \mathrm{a} 184)$ e $3,119 \mathrm{mg} / \mathrm{dl}$ (82 a 161). Os grupos não diferiram entre si quanto aos outros trimestres e quanto à glicemia pós-prandial (GPP) durante toda a gestação. Nenhum grupo atingiu controle glicêmico ideal, mas o perfil glicêmico foi menos adequado no grupo 1 e melhor no grupo 3, embora não tenha havido diferença estatística entre os grupos. Houve um número reduzido de dosagens de 
Tabela 2. Antecedentes clínicos pessoais e familiares analisados nos grupos 1,2 e 3

\begin{tabular}{|c|c|c|c|c|c|c|}
\hline \multirow{2}{*}{ Características } & \multicolumn{2}{|c|}{ DM1 (n:34) } & \multicolumn{2}{|c|}{ DM2 (n:21) } & \multicolumn{2}{|c|}{ DMG (n:38) } \\
\hline & $\mathbf{n}$ & $\%$ & $\mathbf{n}$ & $\%$ & $\mathbf{n}$ & $\%$ \\
\hline \multicolumn{7}{|c|}{ Antecedentes pessoais relevantes } \\
\hline HAS & 2 (a) & 5,9 & 7 (b) & 33,3 & 13 (c) & 34,2 \\
\hline Sobrepeso/Obesidade & 0 (d) & 0,0 & $5(e)$ & 23,8 & $14(\mathbf{f})$ & 36,8 \\
\hline DMG prévio & 0 & 0,0 & 2 & 9,5 & 2 & 5,3 \\
\hline Dislipidemia & 1 & 2,9 & 3 & 14,3 & 1 & 2,6 \\
\hline SOP & 0 & 0,0 & 0 & 0,0 & 1 & 2,6 \\
\hline Tabagismo & $6(\mathbf{g})$ & 17,6 & 11 (h) & 52,4 & $6(\mathbf{i})$ & 15,8 \\
\hline Etilismo & 4 & 11,8 & 1 & 4,8 & 1 & 2,6 \\
\hline Uso de drogas ilícitas & 0 & 0,0 & 0 & 0,0 & 0 & 0,0 \\
\hline $\begin{array}{l}\text { Cetoacidose diabética } \\
\text { prévia }\end{array}$ & $8(\mathbf{j})$ & 23,5 & $0(\mathbf{k})$ & 0,0 & $0(\mathbf{l})$ & 0,0 \\
\hline Hipotireoidismo & 3 & 8,8 & 1 & 4,8 & 0 & 0,0 \\
\hline Cardiopatia isquêmica & $O(\mathbf{m})$ & 0,0 & $2(\mathbf{n})$ & 9,5 & $0(0)$ & 0,0 \\
\hline \multicolumn{7}{|c|}{ Antecedentes familiares relevantes } \\
\hline DM1 & $4(\mathbf{p})$ & 11,8 & $0(\mathbf{q})$ & 0,0 & $0(\mathbf{r})$ & 0,0 \\
\hline DM2 & 20 & 58,8 & 14 & 66,7 & 24 & 63,2 \\
\hline $\mathrm{DMG}$ & 0 & 0,0 & 0 & 0,0 & 0 & 0,0 \\
\hline HAS & 13 & 38,2 & 6 & 28,6 & 8 & 21,0 \\
\hline AVE & 4 & 11,8 & 3 & 14,3 & 2 & 5,3 \\
\hline Cardiopatia isquêmica & 7 & 20,6 & 3 & 14,3 & 8 & 21,0 \\
\hline
\end{tabular}

$\mathbf{a} \times \mathbf{b} \times \mathbf{c}-p=0,009 ; \mathbf{d} \times \mathbf{e} \times \mathbf{f}-p=0,001 ; \mathbf{g} \times \mathbf{h} \times \mathbf{i}-p=0,004 ; \mathbf{j} \times \mathbf{k} \times \mathbf{I}-p=0,001 ; \mathbf{m} \times \mathbf{n} \times \mathbf{0}-p$ $=0,003 ; \mathbf{p} \times \mathbf{q} \times \mathbf{r}-\mathbf{p}=0,027$, sendo que as frequências de $\mathbf{c}, \mathbf{f}, \mathbf{h}$ e j foram maiores que 0 esperado, as de $\mathbf{a}, \mathbf{d}$ e $\mathbf{I}$, menores que o esperado, e de $\mathbf{n}$ e $\mathbf{p}$, com características associadas ao grupo.

HAS: hipertensão arterial sistêmica; SOP: síndrome dos ovários policísticos; AVE: acidente vascular encefálico.

frutosamina e hemoglobina glicosilada durante as gestações analisadas. Quanto às dosagens de frutosamina, tivemos 3 no grupo $1(135,5$ a $215,0 \mu \mathrm{mol} / \mathrm{l}), 6$ no grupo $2(207,0$ a $243,0 \mu \mathrm{mol} / \mathrm{l})$ e 3 no grupo 3 (135,5 a $215,0 \mu \mathrm{mol} / \mathrm{l})$. Quanto às dosagens de hemoglobina glicosilada, tivemos 2 no grupo $1(7,6 \%$ a $9,8 \%), 3$ no grupo $2(6,6 \%$ a $12,2 \%)$ e 2 no grupo $3(7,7 \%$ a $9,8 \%)$. Não foram detectadas diferenças significativas entre os três grupos em relação às concentrações de colesterol total, triglicérides, LDL, HDL, TSH e T4 livre dosados ao longo da gestação (Tabela 3 ).

Analisando separadamente o controle glicêmico das gestantes do grupo 1 , observamos que não houve diferença significativa entre as médias de GJ, GPP e dose diária de insulina NPH no primeiro, segundo e terceiro trimestres de gestação, embora tenha havido queda progressiva dos níveis glicêmicos acompanhada do aumento progressivo da dose de insulina $\mathrm{NPH}$ diária ao longo dos trimestres. A maioria das pacientes fez apli-
Tabela 3. Dados laboratoriais dos grupos 1, 2 e 3, obtidos antes e durante a gestação

\begin{tabular}{|c|c|c|c|}
\hline \multirow{2}{*}{ Características } & \multicolumn{3}{|c|}{ Grupos clínicos } \\
\hline & DM1 & DM2 & DMG \\
\hline \multicolumn{4}{|c|}{ Dados laboratoriais pré-gestacionais* } \\
\hline Glicemia de jejum (mg/dl) & $192(88-318)$ & $146(93-278)$ & - \\
\hline Glicose pós-prandial (mg/dl) & $217(113-502)$ & $188(121-456)$ & - \\
\hline Frutosamina $(\mu \mathrm{mol} / \mathrm{l})$ & $458(288-757)$ & $297(225-404)$ & - \\
\hline HbA1c (\%) & $12(10-14)$ & 6,35 & - \\
\hline Colesterol total (mg/dl) & $174(138-225)$ & $172(136-188)$ & - \\
\hline LDL colesterol (mg/dl) & $90(44-151)$ & $85,5(60-100)$ & - \\
\hline HDL colesterol (mg/dl) & $63(30-75)$ & $49(32-78)$ & - \\
\hline Triglicérides (mg/dl) & $76(27-162) \mathbf{a}$ & $154(145-291) \mathbf{b}$ & - \\
\hline \multicolumn{4}{|c|}{ Dados laboratoriais durante a gestação } \\
\hline \multicolumn{4}{|l|}{ Glicemia de jejum (mg/dl) } \\
\hline $1^{\circ}$ trimestre & $172(77-358) \mathbf{c}$ & $115(97-184) \mathbf{d}$ & $119(82-161)$ \\
\hline $2^{\circ}$ trimestre & $132(36-212)$ & $126(84-192)$ & $104(82-172)$ \\
\hline $3^{\circ}$ trimestre & $113(65-189)$ & $110(81-270)$ & $104(69-173)$ \\
\hline \multicolumn{4}{|l|}{ Glicose pós-prandial (mg/dl) } \\
\hline $1^{\circ}$ trimestre & $181(111-213)$ & $165(115-257)$ & $150(148-184)$ \\
\hline $2^{\circ}$ trimestre & $168(100-287)$ & 151 (101-251) & $149(118-230)$ \\
\hline $3^{\circ}$ trimestre & $136(76-237)$ & $145(101-303)$ & $135(66-199)$ \\
\hline Colesterol total (mg/dl) & $197(141-261)$ & $189(76-277)$ & $156(102-331)$ \\
\hline Triglicérides (mg/dl) & $167(66-221)$ & $168(70-336)$ & $182(112-362)$ \\
\hline LDL colesterol (mg/dl) & $137(87-150)$ & $133(84-159)$ & $67(40-131)$ \\
\hline HDL colesterol (mg/dl) & $60(32-73)$ & $51(37-76)$ & $51(40-74)$ \\
\hline $\mathrm{HbA1c}(\%)$ & $13(10-17)$ & $7(6-12)$ & $9(8-10)$ \\
\hline Frutosamina $(\mu \mathrm{mol} / \mathrm{l})$ & $283(139-513)$ & $221(207-243)$ & $205(135-215)$ \\
\hline TSH (ng/dl) & $1,1(0,4-4,8)$ & $1,4(0,3-2,9)$ & $1,2(0,1-5,9)$ \\
\hline T4 livre (ng/dl) & $1,1(0,8-1,7)$ & $1,0(0,8-1,5)$ & $1,0(0,8-2,3)$ \\
\hline
\end{tabular}

$\mathbf{a} \times \mathbf{b}(a<b)-p=0,005 ; \mathbf{c} \times \mathbf{d}(c>d)-p=0,027$.

* Média dos 3 últimos anos.

cações de insulina NPH divididas em duas (dois terços antes do café da manhã e um terço antes do jantar ou ao deitar-se) ou até três doses diárias, com correções em bolus das hiperglicemias pré-alimentares com insulina regular; como essas doses variaram dia a dia, para efeito de análise estatística, foram consideradas apenas as doses de insulina de ação intermediária.

Nas gestantes do grupo 2, não houve diferença significativa entre as médias de GJ e GPP no primeiro, segundo e terceiro trimestres, mas houve diferença significativa $(\mathrm{p}<0,001)$ quanto à dose média de insulina $\mathrm{NPH}$ diária necessária em cada trimestre, havendo aumento progressivo do início ao fim da gestação. Entre essas pacientes, seis $(28,6 \%)$ estavam fazendo uso de hipoglicemiante(s) oral(is) no início da gestação, sendo 
Tabela 4. Análise do peso e IMC dos grupos 1, 2 e 3 durante a gestação*

\begin{tabular}{|c|c|c|c|}
\hline & & Peso (kg) & IMC $\left(\mathrm{kg} / \mathrm{m}^{2}\right)$ \\
\hline \multirow[t]{3}{*}{ DM1 } & $1^{0} \mathrm{~T}$ & $59(42-76) \mathbf{a}$ & $23(17-27) \mathbf{d}$ \\
\hline & $2^{0} \mathrm{~T}$ & $64(47-79) \mathbf{b}$ & $26(19-29) \mathbf{e}$ \\
\hline & $3^{0} \mathrm{~T}$ & $70(55-93) \mathbf{c}$ & $27(22-33) \mathbf{f}$ \\
\hline \multirow[t]{3}{*}{ DM2 } & $1^{0} \mathrm{~T}$ & $69(53-103) \mathbf{g}$ & $29(22-38) \mathbf{j}$ \\
\hline & $2^{0} \mathrm{~T}$ & $73(57-123) \mathbf{h}$ & $31(23-45) \mathbf{k}$ \\
\hline & $3^{\circ} \mathrm{T}$ & $77(60-128) \mathbf{i}$ & $31(23-47)$ I \\
\hline \multirow[t]{3}{*}{ DMG } & $1^{0} \mathrm{~T}$ & $77(44-90) \mathbf{~ m}$ & $32(18-36) \mathbf{p}$ \\
\hline & $2^{0} \mathrm{~T}$ & $85(49-136) \mathbf{n}$ & $34(20-53) \mathbf{q}$ \\
\hline & $3^{0} \mathrm{~T}$ & $88(30-139)$ o & $36(22-54) \mathbf{r}$ \\
\hline
\end{tabular}

$\mathbf{a} \times \mathbf{b} \times \mathbf{c}(\mathrm{a}<\mathrm{b}<\mathrm{c}): \mathrm{p}<0,001 ; \mathbf{d} \times \mathbf{e} \times \mathbf{f}(\mathrm{d}<\mathrm{e}<\mathrm{f}): \mathrm{p}<0,001 ; \mathbf{g} \times \mathbf{h} \times \mathbf{i}(\mathrm{g}<\mathrm{h}<\mathrm{i}): \mathrm{p}=0,002 ;$ $\mathbf{j} \times \mathbf{k} \times \mathbf{I}(j<k<\mathrm{l}): p=0,002 ; \mathbf{m} \times \mathbf{n} \times \mathbf{0}(m<n<0): p=0,004 ; \mathbf{p} \times \mathbf{q} \times \mathbf{r}(p<q<r): p=0,38$.

* Valores expressos em mediana (mínimo-máximo); T: trimestre.

que uma fazia uso de metformina (interrompida com IG de 20 semanas), uma de metformina associada a glibenclamida (interrompidas com IG de 8 semanas), três de glibenclamida isoladamente (interrompida entre $9 \mathrm{e}$ 13 semanas de gestação), uma de glibenclamida associada a clorpropamida (interrompidas com 17 semanas de gestação).

No grupo 3, também não observamos diferença significativa entre as médias de GJ e GPP no primeiro, segundo e terceiro trimestres, mas houve maior necessidade de insulina NPH $(\mathrm{p}<0,001)$ no terceiro trimestre em comparação com o primeiro e segundo trimestres. Ainda neste grupo observamos que $26(68,4 \%)$ das pacientes perderam o acompanhamento após o parto e, entre as mulheres que mantiveram o seguimento $(\mathrm{n}=$ 12), 11 (91,6\%) evoluíram para DM2.

Todas as pacientes foram controladas com dieta e insulina no esquema basal (NPH) de duas a três vezes ao dia, bolus (insulina regular) antes das refeições com períodos mais intensos de monitorização glicêmica em regime hospitalar. Os três grupos não diferiram quanto à adequação alimentar considerando-se ingestão proteico-calórica. Quanto às doses de insulina, foram analisadas as médias de unidades de $\mathrm{NPH} /$ dia e em cada trimestre, tendo sido observada diferença $(\mathrm{p}<$ $0,001)$ entre os três grupos no segundo trimestre, entre o grupo 1 e os demais no primeiro trimestre e entre os grupos 1 e 3 no terceiro trimestre $(\mathrm{p}=0,004)$. Constatamos necessidade maior de insulina nos pacientes com DMl ao longo de toda a gestação e necessidade menor em pacientes com DMG. Não houve diferença significativa quanto ao tempo de internação para controle glicêmico entre os três grupos. Durante a gestação analisada, houve predomínio de cetoacidose diabética no grupo $1(\mathrm{n}=12 ; \mathrm{p}<0,001)$.
Considerando o tipo de parto, não houve diferença entre os três grupos, predominando cesariana (Tabela l). As complicações do parto estão expressas na tabela 1 . No grupo 1 , neonatos apresentaram mais sinais de tocotrauma $(\mathrm{p}=0,023)$ e foi maior o número de prematuros, embora não tenha existido diferença estatisticamente significativa entre os grupos. Pacientes do grupo 2 apresentaram maior incidência de infecção tardia da ferida operatória $(\mathrm{p}=0,03)$.

As complicações fetais foram expressas na tabela 5 . O desconforto respiratório foi, no geral, a ocorrência mais frequente $(\mathrm{n}=21,22,6 \%)$, principalmente no grupo 1 , mas não diferiu significantemente entre os grupos analisados. Não observamos diferença quanto à ocorrência de hipoglicemia fetal $(\mathrm{n}=20 ; 21,5 \%)$, quanto ao peso dos neonatos dos grupos 1, 2 e 3 , cujas medianas e variações foram $3.460 \mathrm{~g}$, (1.120 a 4.665), $3.245 \mathrm{~g}$ (680 a 4.615) e $3.500 \mathrm{~g}$ (795 a 5.330), respectivamente, e quanto ao tempo de seguimento materno após o parto nos grupos 1,2 e 3 , cujas medianas e variações foram de 3 anos $(0,2$ a 10), 3 (1 a 13) e $4(0,5$ a 15$)$, respectivamente.

Tabela 5. Avaliação clínica e metabólica dos neonatos de mães portadoras de diabetes melito tipo 1 (G1), tipo 2 (G2) e gestacional (G3)

\begin{tabular}{|c|c|c|c|c|c|c|}
\hline \multirow[t]{2}{*}{ Caracteristicas } & \multicolumn{2}{|c|}{$\begin{array}{c}\text { DM1 } \\
(n=34)\end{array}$} & \multicolumn{2}{|c|}{$\begin{array}{c}\text { DM2 } \\
(n=21)\end{array}$} & \multicolumn{2}{|c|}{$\begin{array}{c}\text { DMG } \\
(n=38)\end{array}$} \\
\hline & $\mathbf{n}$ & $\%$ & $\mathbf{n}$ & $\%$ & $\mathbf{n}$ & $\%$ \\
\hline Hipoglicemia fetal & 9 & 26,5 & 4 & 19,0 & 7 & 18,4 \\
\hline \multicolumn{7}{|c|}{ Malformações/Complicações fetais } \\
\hline Macrossomia & 3 & 8,8 & 0 & 0,0 & 5 & 13,2 \\
\hline Desconforto respiratório & 11 & 32,3 & 4 & 19,0 & 6 & 15,8 \\
\hline Cardiopatias & 2 & 5,9 & 0 & 0,0 & 2 & 5,3 \\
\hline $\begin{array}{l}\text { Malformações } \\
\text { musculoesqueléticas }\end{array}$ & 2 & 5,9 & 1 & 4,8 & 2 & 5,3 \\
\hline Icterícia & 2 & 5,9 & 0 & 0,0 & 2 & 5,3 \\
\hline Onfalocele/gastrosquise & 0 & 0,0 & 0 & 0,0 & 1 & 2,6 \\
\hline $\begin{array}{l}\text { Hérnia diafragmática } \\
\text { congênita }\end{array}$ & 1 & 3,4 & 0 & 0,0 & 0 & 0,0 \\
\hline Atresia duodenal & 1 & 3,4 & 0 & 0,0 & 0 & 0,0 \\
\hline Natimorto & 2 & 5,9 & 2 & 9,5 & 1 & 2,6 \\
\hline
\end{tabular}

\section{DISCUSSÃO}

Em geral, gestantes que desenvolvem DG são diagnosticadas nos serviços de GO quando rastreadas no segundo trimestre e o tratamento transcorre sem a participação dos serviços de Endocrinologia. Não raro, a gestante sequer faz pré-natal e procura assistência tardiamente, sendo o DG diagnosticado no período próxi- 
mo ao parto. Por outro lado, os serviços de Endocrinologia aconselham pacientes portadoras de DM1 e DM2 a engravidarem com controle glicêmico adequado e a retornarem no período imediato do diagnóstico de gestação, o que nem sempre acontece.

O diferencial deste estudo é que avaliamos gestantes que foram assistidas pela disciplina de Endocrinologia em conjunto com a disciplina de $\mathrm{GO}$, entre as quais a maioria necessitou de internações durante a gestação para adequar o controle glicêmico visando à melhor evolução materno-fetal. Trata-se de um grupo selecionado de pacientes, sendo grande parte $(\mathrm{n}=55)$ proveniente do ambulatório de diabetes (DM1 e DM2); todas as portadoras de DG necessitaram de assistência para o controle glicêmico, obtido mediante insulinização, e foram à primeira consulta endócrina no tempo adequado (mediana de 27 semanas).

Em relação à idade, as portadoras de DMl foram as mais jovens e as com DM2 tiveram idade materna avançada, refletindo a idade de aparecimento do DM e o aconselhamento médico da equipe às portadoras de DMl para engravidarem antes do aparecimento de complicações.

A gestação analisada pelo estudo nem sempre foi a primeira e as ocorrências prévias mais encontradas que poderiam complicar o parto, fetos macrossômicos, eclâmpsia e pré-eclâmpsia foram mais frequentes nas portadoras de DG e DM2, respectivamente (Tabela 1), indicando que as condições de risco para a gestação atual, como obesidade e hipertensão arterial, preexistiram e não foram corrigidas num planejamento gestacional adequado com análise dos fatores de risco $(5,20,21)$.

Com relação aos antecedentes pessoais, a única ocorrência especial e relevante $(\mathrm{p}<0,05)$ no grupo $\mathrm{l}$ foi, como se esperaria, a existência de episódios prévios de cetoacidose diabética. Os grupos 2 e 3 apresentaram semelhanças entre si, diferindo em relação ao grupo 1 (Tabela 2). Hipertensão arterial, sobrepeso e obesidade foram mais encontrados no grupo 3 (DG) do que até mesmo no grupo 2 (DM2) $(\mathrm{p}<0,05)$. A prevalência de tabagismo não diferiu nos três grupos e foi elevada $(24,3 \%)$, indicando uma vez mais o planejamento gestacional inadequado e presença de mais um fator de risco para complicações fetais $(20)$.

Como era esperado, antecedentes de DMl não foram encontrados nos grupos 2 e 3 e apenas em 11,8\% do próprio grupo $1(n=4)$. Entretanto, foi elevada a prevalência de DM2 em todos os grupos, bem como de hipertensão arterial e cardiopatia isquêmica (Tabela 2).
Segundo a literatura $(7,22,23)$, a incidência de DG duplicou nos últimos 8 anos, em paralelo com a epidemia de obesidade, com risco elevado de desenvolvimento do DM2 na mãe e na prole. A resistência insulínica é preexistente e piora com a gestação. Além disso, mulheres com história de DG têm um risco que varia de $50 \%$ a $80 \%$ de desenvolver DM2, sendo questionado por alguns autores se não se trata da mesma doença em diferentes tempos de evolução $(6,24,25)$. Os antecedentes pessoais e familiares dos grupos 2 e 3 sugerem a existência de bases genéticas e ambientais comuns (8). A elevada prevalência de DM2 nos antecedentes do grupo 1 merece uma investigação à parte na comunidade estudada.

$\mathrm{O}$ controle glicêmico prévio à gestação analisada foi avaliado nos três anos anteriores por meio da média aritmética dos valores das glicemias de jejum e pósprandial, bem como da frutosamina e hemoglobina glicada. No geral, os pacientes diabéticos retornam a cada 3 a 4 meses e a opção pela média dos últimos três anos implicou a realização de médias entre 12 determinações glicêmicas. Embora as diferenças não sejam significativas, o controle glicêmico mostrou uma tendência em ser pior no grupo 1, enquanto o controle lipídico, sobretudo com relação aos triglicérides $(\mathrm{p}<0,05)$, foi pior no grupo 2 (Tabela 3 ). Tais dados explicam a maior ocorrência de complicações do DM no grupo 1 (sobretudo retinopatia, em 20,6\%), pois a duração da doença foi semelhante entre os grupos 1 e 2 . Não se observou, contudo, piora evolutiva das complicações no grupo l e uma possível justificativa positiva é que o controle glicêmico melhorou ao longo da gestação analisada (Tabela 3), ressaltando a importância do controle glicêmico e sua relação com a evolução das complicações, como já demonstrado por outros autores $(26,27)$.

As gestantes dos grupos 2 e 3 apresentaram sobrepeso/obesidade e, consequentemente, IMC elevado já no primeiro trimestre, em relação ao grupo 1 , e essa diferença persistiu durante toda a gestação $(\mathrm{p}<0,05)$. Não obstante as pacientes referirem adesão à orientação nutricional recebida da nutricionista e do médico-assistente, segundo recomendações atuais (28), não foi analisada a ingestão de macro e micronutrientes por julgarmos inviável obter esses dados numa análise retrospectiva.

As médias glicêmicas do primeiro trimestre foram maiores no grupo 1 em relação ao 2 , mas no segundo e no terceiro trimestres foram semelhantes nos três grupos. Embora nenhum grupo tenha atingido o controle adequado e ideal (29-31), o controle glicêmico foi melhorando no decorrer da gestação em relação ao primeiro 
trimestre e ao controle pré-gestacional, à custa de internações hospitalares para adequação de doses crescentes de insulina. Para fins de cálculo estatístico, consideramos apenas as doses de NPH introduzida mais precocemente no grupo 2 do que no 3 . Entretanto, as correções préprandiais foram feitas pelos bolus de insulina regular. Não foi possível quantificar o controle glicêmico mediante as dosagens de frutosamina e hemoglobina glicada em razão do pequeno número de determinações resgatadas dos prontuários. Como tais dados não se prestam a fornecer informações fidedignas durante a gestação e porque não podem ser tomados como parâmetros para modificar a terapêutica $(32,33)$, houve preferência, em nosso meio, em analisar o perfil glicêmico diário (constituído por cinco determinações de glicemias capilares/dia) e ir enfrentando o desafio de corrigir hiperglicemias pré e pós-prandiais com insulina regular orientando as pacientes em retornos mais frequentes para análise dos registros de automonitoramento domiciliar e internações hospitalares quando o controle glicêmico se tornava inaceitável.

No grupo 2, 6 pacientes $(28,5 \%)$ usaram hipoglicemiantes orais no período da organogênese e foram orientadas a interromper a medicação quando iniciado o seguimento médico da gravidez. A partir do diagnóstico de gravidez, os hipoglicemiantes orais foram substituídos por insulina. Apesar de alguns estudos mostrarem que estes poderiam ser uma alternativa viável durante a gestação $(34,35)$, em nosso meio a substituição pela insulina ainda é a conduta padrão.

A presença de diabetes, por si só, não é uma indicação de parto cesariana (36), entretanto, em decorrência da macrossomia fetal, da elevada frequência de distocia de ombro com lesão de plexo braquial e das complicações maternas como laceração perineal e incontinência urinária, alguns obstetras têm recomendado parto cesariana em mulheres diabéticas (36-38), embora sejam necessários mais estudos controlados a esse respeito. Nesta casuística, observamos opção preferencial pela cesariana, sobretudo em razão das frequentes complicações do parto referidas na tabela 1 e que foram mais frequentes no grupo 1.

Outras complicações observadas (Tabela 1) foram mais frequentes no grupo 1 , embora do ponto de vista estatístico apenas o tocotraumatismo foi maior neste grupo $(\mathrm{p}<0,05)$ e infecção da ferida operatória só foi encontrada no grupo 2 , indicando maior morbidade do parto de portadoras de DMl.

$\mathrm{Na}$ escolha do melhor momento para a interrupção do parto, deve-se sempre levar em conta o risco e bene- fício para a mãe e o feto (36). Nesta casuística, partos pré-termo e natimortalidade estiveram relacionados à ocorrência de eclâmpsia e pré-eclâmpsia e à amniorrexe prematura. A literatura tem relacionado esses eventos à obesidade, à hipertensão arterial, bem como à piora do controle glicêmico no último trimestre de gestação (36-42). As portadoras de DMl não apresentaram obesidade, mas complicações do DM, como nefropatia e hipertensão arterial, e pior perfil glicêmico no primeiro trimestre. Nos outros grupos, a obesidade e a hipertensão arterial seguramente contribuíram para esses eventos, como sugere a literatura.

As complicações fetais foram mais frequentes no grupo 1 , embora os percentuais apenas tenham atingido nível de significância $(\mathrm{p}<0,05)$ em frequência de tocotraumatismo em relação aos grupos 2 e 3 (Tabela 5). Ainda assim, a maioria dos recém-nascidos sobreviveu, sendo baixa a frequência de natimortos $(\mathrm{n}=5)$ distribuída igualmente entre os três grupos, o que justifica o esforço da equipe incluindo a assistência do grupo de neonatologia e terapia intensiva infantil. A hipoglicemia fetal foi igualmente elevada nos três grupos e esses dados são semelhantes à literatura e atribuídos ao controle glicêmico inadequado (36-42).

Analisando os dados deste estudo como um todo, podemos concluir que a maioria das gestações em diabéticas, conduzidas em nosso meio, evoluiu favoravelmente, tanto para mãe como para o feto. A melhor adequação do controle glicêmico poderá reduzir ainda mais a frequência de hipoglicemia neonatal e as outras complicações dos recém-nascidos nessas condições (43). Essa análise abrange um período em que pacientes diabéticas não tinham acesso à automonitoração financiada pelo Sistema Único de Saúde (SUS). Nos últimos anos, uma lei federal definiu como obrigação do Estado o fornecimento de insumos para o controle glicêmico de pacientes com DM, e isso deve também facilitar o controle do DG e da gestação em portadoras de DM1 e DM2 que necessitam de automonitoração intensiva para correções frequentes das concentrações glicêmicas durante toda a gestação (44).

O seguimento das portadoras de DG também deve ser melhorado por meio da orientação e conscientização materna da necessidade de prevenção do DM2. No grupo estudado, o seguimento pós-parto foi feito em apenas 12 pacientes e, destas, 11 (91,6\%) evoluíram para DM2 (Tabela 4), mostrando que o DG é apenas a antecipação do DM2 em consequência das condições gestacionais capazes de desencadear o diabetes, como 
maior ganho de peso, resistência insulínica, menor atividade física, entre outros fatores, e que a prevenção do DM2 poderia ser feita imediatamente após o parto, assim que os níveis glicêmicos retornam ao normal (45).

Agradecimentos: À equipe de médicos-residentes e professores da disciplina de Endocrinologia do HC-UFTM que assistiram as pacientes ao longo do período analisado e à professora de língua inglesa Ângela Maria Fraga Azôr, que contribuiu com a tradução do resumo deste artigo.

Declaração: os autores declaram não haver conflitos de interesse científico neste estudo.

\section{REFERÊNCIAS}

1. Amos A, McCarty DJ, Zimmet P. The rising global burden of diabetes and its complication: estimates and projections for 2003. Diabet Med. 1997;Suppl 5:S1-85.

2. Wild S, Roglic G, Green A, Sicree R, King H. Global prevalence of diabetes. Estimates for the year 2000 and projections for 2030 . Diabetes Care. 2004;27:1047-53.

3. The Expert Committee on the Diagnosis and Classification of Diabetes Mellitus. Report of the Expert Committee on the Diagnosis and Classification of Diabetes Mellitus. Diabetes Care. 2000;23 Suppl 1:S4-19.

4. Lucas MJ. Diabetes complicating pregnancy. Obstet Gynecol Clin North Am. 2001;28:513-36.

5. Mulholland C, Njoroge T, Mersereau RN, Williams J. Comparison of guidelines available in the United States for diagnosis and management of diabetes before, during, and after pregnancy. $J$ Womens Health. 2007;16:790-801.

6. Langer 0 . Management of gestational diabetes: pharmacologic treatment options and glycemic control. Endocrinol Metab Clin North Am. 2006;35:53-78.

7. Ferrara A. Increasing prevalence of gestational diabetes. Diabetes Care. 2007;30:141-46.

8. BRASIL. Ministério da Saúde. Secretaria de Atenção à Saúde. Departamento de Atenção Básica. Coordenação Geral da Política de Alimentação e Nutrição. Vigilância Alimentar e Nutricional-Sisvan. Série A. Normas e Manuais Técnicos 2004. Disponível em: http://dab.saude.gov.br/nutrícao/publicacoes.php\#sisvan. Acesso em: 13 Mar 2010.

9. American Diabetes Association. Diagnosis and classification of diabetes mellitus. Clinical practice recommendations. Diabetes Care. 2004;27 Suppl 1:S5-10.

10. American Diabetes Association. Clinical practice recommendations. Gestational Diabetes. Diabetes Care. 2001;24 Suppl 1:S77-9.

11. American Diabetes Association. Standards of Medical Care in Diabetes - 2010. Diabetes Care. 2010;Suppl 1:S11-61.

12. Summary and Recommendations of the Fith International Workshop-Conference on Gestation Diabetes Mellitus. Diabetes Care. 2007;Suppl 2:S251-60.

13. Kunst A, Draeger B, Ziegenborn J. Colorimetric methods with glucose oxidase and peroxidase. In: Bergemeyer HU. 3rd ed. Methods of enzymatic analysis. Metabolites I. Carbohydrates. Verlag-Chemie. 1988; 4:163-72.

14. Zander R, Lang W, Wolf HV. Alkaline haematin O-575, a new tool for the determination of haemoglobin as an alternative to the cyanohemoglobin method. I. Description of the method. Clin Chem Acta. 1984;136:83-93.
15. Kruse-Jarres JD, Jaraush J, Lehmann P, Vogt BW, Rietz P. A new colorimetric method for the determination of fructosamine. Lab Med. 1989;13:245-53.

16. Tietz NW. Clinical guide to laboratory tests. 3rd ed. Philadelphia PA: WB Saunders Company. 1995;130-31.

17. Fossati $P$, Principle L. Serum triglycerides determined colorimetrically with an enzyme that produces hydrogen peroxide. Clin Chem. 1982;28:2077-80.

18. MatsuzakiY, Kawaguchi E, Morita, et al. Evaluation of two kinds of reagents for direct determination of HDL-colesterol. J Anal Bio-Sc. 1996;19:419-27.

19. Bronstein I, Juo RR, Voyta JC. Novel chemiluminescent adamantly 1,2 dioxetane enzyme substrate. In: Stanley P, Krisha LJ. Bioluminescence and Chemiluminescence. England: John Wiley \& Son. Chichester. 1991; p. 74-82.

20. Rudra CB, Sorensen TK, Leisering WM, Dashow E, Williams MA. Weight characteristics and height in relation to risk of gestational diabetes mellitus. Am J of Epidemiol. 2007;165:302-8.

21. Dode MASO, Santos IS. Non classical risk factors for gestational diabetes mellitus: a systematic review of the literature. Cad Saúde Pública. 2009;Suppl 3:S341-59.

22. Barbour LA, McCurdy CE, Hernandez TL, Kirwan JP, Catalano $P M$, Friedman JE. Celular mechanisms for insulin resistence in normal pregnancy and gestational diabetes. Diabetes Care. 2007;30:112-9.

23. McLean M, Chipps D, Cheung NW. Mother to child transmission of diabetes mellitus: does gestational diabetes program type 2 diabetes in the next generation? Diabetic Medicine. 2006;23:1213-15.

24. Pendergrass E, Fazioni E, DeFronzo RA. Non-insulin dependent diabetes mellitus and gestational diabetes mellitus: same disease, another name? Diabetes Rev. 1995;3:584-601.

25. Watanabe $\mathrm{R}, \mathrm{Black} \mathrm{MH}$, Xiang $\mathrm{AH}$, Allayee $\mathrm{HA}$, Lawrence $\mathrm{JH}$, Buchanan TA. Genetics of gestational diabetes mellitus and type 2 diabetes. Diabetes Care. 2007;30:134-40.

26. Chen R, Ben-Haroush A, Weissmann-Brenner A, Melamed N, Hod M, Yogev $Y$. Levels of glycemic control and pregnancy outcome in type 1 diabetes: a comparison between multiple daily insulin injections and continuous insulin infusions. Am J Obstet Gynecol. 2007;197:404.e1-5.

27. Krstevska B, Mishevska S, Janevska E, Simeonova S, Livrinova V, Pemovska G, et al. Gestational diabetes mellitus - the impact of maternal body mass index and glycaemic control on baby's birth weight. Prilozi. 2009;30(2):115-24.

28. Artal R, Lockwood CJ, Brown HL. Weight gain recommendations in pregnancy and th obesity epidemic. Obstet Gynecol. 2010;115(1):152-55.

29. Hollander MH, Paarlberg KM, Huisjes AJM. Gestational diabetes: a review of the current literature and guidelines. Obstet Gynecol Surv. 2007;62:125-36.

30. Renard E, Raingeard I, Boulot P, Bringer J. Insulin therapy in type 1 diabetes for and during pregnancy: by which means and for which objectives? Diabetes Metab. 2001;27(4 Pt 2):S61-6.

31. Kinsley B. Achieving better outcomes in pregnancies complicated by type 1 and type 2 diabetes mellitus. Clin Ther. 2007;29 Suppl D:S153-60.

32. Hashimoto K, Noguchi S, MorimotoY, Hamada S, Wasada K, Imai S, et al. A1c but not serum glycated albumin is elevated in late pregnancy owing to iron deficiency. Diabetes Care. 2008;31:1945-48.

33. Paroni R, Ceriotti F, Galanelo R, Leoni GB, Panico A, Scuratti E, et al. Performance characteristics and clinical utility of an enzymatic method for the measurement of glycated albumin in plasma. Clin Biochem. 2007;40:1398-405.

34. Homko CJ, Reece A. Insulins and oral hypoglycemic agents in pregnancy. J Matern fetal Neonatal Med. 2006;19:679-86. 
35. Silva J, Bertini AM, Taborda W, Becker F, Bebber FRL, Aquim GDMC, et al. Glibenclamida no tratamento do diabete melito gestacional em estudo comparado à insulina. Arq Bras Endocrinol Metab. 2007;51/4:541-6.

36. Hawkins JS, Casey BM. Labor and delivery management for women with diabetes. Obstet Gynecol Clin North Am. 2007;34:323-34.

37. Ben-Haroush A, Hadar E, Chen R, Hod M, Yogev Y. Maternal obesity is a major risk factor for large-for-gestational infants in pregnancies complicated by gestational diabetes. Arch Gynecol Obstet. 2008;279:539-43.

38. Mansor A, Arumugam K, Omar SZ. Macrosomia is the only reliable predictor of shoulder dystocia in babies weighing $3.5 \mathrm{~kg}$ or more. Eur J Obstet Gynecol Reprod Biol. 2010;149(1):44-6.

39. Conway D. Obstetric management in gestational diabetes. Diabetes Care. 2007;30Suppl 2:S175-79.

40. Campos MV, Ruas L, Paiva S, Leitão P, Lobo C, Marta E, et al. Crescimento fetal e controlo glicémico em grávidas diabéticas tipo 1 . Acta Med Port. 2004;17:167-72.
41. González-Quintero VH, Istwan NB, Rhea DJ, Rodriguez LI, Cotter A, Carter $\mathrm{J}$, et al. The impact of glycemic control on neonatal outcome in singleton pregnancies complicated by gestational diabetes mellitus. Diabetes Care. 2007;30:467-70.

42. Lauenborg J, Mathiesen E, Ovesen P, Westergaard JG, Ekbom P, Molsted-Pedersen $\mathrm{L}$, et al. Audit on stillbirths in women with pregestational type 1 diabetes. Diabetes Care. 2003;26: 1385-89.

43. Horvath K, Koch K, Jeitler K, Matyas E, Bender R, Bastian H, et al. Effects of treatment in women with gestational diabetes mellitus: systematic review and meta-analysis. BMJ. 2010;340:1-18.

44. Silva LIL, Bastos MT, Mantega G, Silva JB Jr. Lei $n^{\circ} 11.347$, de 27 de setembro de 2006. Brasília, DF. 2006. Disponível em: http:// www.brasilsus.com.br/legislacoes/leis/101534-11347. Acesso em: 28 Mar 2010.

45. Retnakaran R, Connelly PW, Sermer M, Zinman B, Hanley AJG. The impact of family history of diabetes on risk factors for gestational diabetes. Clin Endocrinol. 2007;67(5):754-60. 